Exercices Exercices de rhétorique

de rhétorique $15 \mid 2020$

Sur (et contre) les figures

\title{
Apologie pour la rhétorique [1890]
}

Notice et notes: Ilaria Vidotto

Ferdinand Brunetière

Ilaria Vidotto (éd.)

\section{(2) OpenEdition}

Journals

Édition électronique

URL : http://journals.openedition.org/rhetorique/1076

DOI : 10.4000/rhetorique.1076

ISSN : 2270-6909

Éditeur

UGA Éditions/Université Grenoble Alpes

Édition imprimée

ISBN : 978-2-37747-248-2

\section{Référence électronique}

Ferdinand Brunetière, «Apologie pour la rhétorique [1890] », Exercices de rhétorique [En ligne], 15 |

2020, mis en ligne le 07 novembre 2020, consulté le 03 décembre 2020. URL : http://

journals.openedition.org/rhetorique/1076 ; DOI : https://doi.org/10.4000/rhetorique.1076

Ce document a été généré automatiquement le 3 décembre 2020.

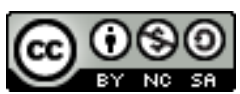

Les contenus de la revue Exercices de rhétorique sont mis à disposition selon les termes de la Licence Creative Commons Attribution - Pas d'Utilisation Commerciale - Partage dans les Mêmes Conditions 4.0 International. 


\title{
Apologie pour la rhétorique [1890]
}

\author{
Notice et notes: Ilaria Vidotto
}

\section{Ferdinand Brunetière}

llaria Vidotto (éd.)

\section{Notice}

1 Ferdinand Brunetière publie son «Apologie de la rhétorique » dans la Revue des Deux Mondes du $1^{\mathrm{er}}$ décembre 1890. Collaborateur de proue depuis les années 1870 de cette même revue, dont il assumera la direction en $1893^{1}$, Brunetière allie à son métier de critique et d'historien de la littérature une tâche d'enseignement à l'École normale supérieure, où il occupe à partir de 1886 la fonction de maître de conférences. À l'époque où est livré le texte que nous éditons ici, Brunetière a déjà fait paraître, outre de très nombreux articles dans La Revue des deux Mondes, deux grands recueils d'études critiques (Histoire et littérature, 3 vol., Calmann-Lévy, 1884-1886; Questions et Nouvelles questions de critique, Calmann-Lévy, 1889-1890), plusieurs volumes de ses Études critiques sur l'histoire de la littérature française (Hachette), dont la publication s'échelonne de 1880 à 1903, ainsi que L'Évolution de la critique depuis la Renaissance jusqu'à nos jours (Hachette, 1890), ouvrage dans lequel Brunetière développe sa célèbre théorie de l'évolution des genres littéraires inspirée de la doctrine darwinienne.

Dans les débats qui traversent, en ce $\mathrm{XIX}^{\mathrm{e}}$ siècle finissant, la République des lettres, Brunetière se distingue par ses opinions résolument conservatrices, voire réactionnaires. Sur le plan de la critique littéraire, il fustige les esthétiques contemporaines et juge sévèrement les productions les plus marquantes de son temps. Ses foudres n'épargnent ni le roman naturaliste et son chef de file Zola, accusés de matérialisme et de grossièreté ${ }^{2}$, ni la poésie parnassienne et surtout Baudelaire, « mystificateur ${ }^{3}$ » amoral et outrancier, dont l'influence corruptrice pervertit les jeunes poètes contemporains. À l'égard des « symbolistes et décadents » qui dominent la scène littéraire de la fin du siècle, Brunetière tient des propos ambivalents : s'il blâme, d'une part, les excès langagiers et l'individualisme des jeunes littérateurs, tout comme leur absence de culture littéraire - qui leur fait prendre pour modèles, au lieu des grands 
maîtres de la tradition, des auteurs sans légitimité -, il leur accorde, d'autre part, le mérite d'avoir réintroduit dans leurs écrits des problématiques d'ordre spirituel et métaphysique, complètement évincées par la doctrine naturaliste ${ }^{4}$.

3 Sur un plan institutionnel, les campagnes de réforme entreprises aux alentours des années 1880-1900 dans le domaine de l'enseignement secondaire et supérieur du français suscitent des réactions tout aussi anti-progressistes de la part du pédagogue Brunetière. Toujours depuis les colonnes de la Revue des deux mondes, ce promoteur de l'histoire littéraire défend en 1891 une conception de l'enseignement de la littérature tournée vers le passé. Il invoque, à ce propos, le bannissement des œuvres contemporaines des programmes officiels, dont le soubassement doit être constitué uniquement par un ensemble de textes de l'époque classique, formant la base de connaissances indispensable au maintien de la tradition française ${ }^{5}$. Mais c'est surtout vis-à-vis de la rhétorique que Brunetière prend, en véritable "arrière-gardiste", le contrepied de l'opinion ambiante. À l'heure où les réformateurs préconisent un enseignement $d u$ français de plus en plus affranchi de l'emprise de la tradition rhétorique, et où se multiplient les attaques portées contre cet « art sans profondeur, qui ne s'occupe et ne traite que de l'arrangement des mots et de l'arrangement des phrases $^{6} "$, Brunetière non seulement défend la place de la rhétorique dans l'enseignement supérieur, en tant que branche de l'histoire de la langue ${ }^{7}$, mais s'applique plus largement à en illustrer la permanence, ainsi que le caractère consubstantiel à la pensée et à l'écriture - des thèses qui seront reprises, au milieu du siècle suivant, par Jean Paulhan dans Les Fleurs de Tarbes ou la Terreur dans les lettres.

4 Ce rapide aperçu des positions idéologiques de Brunetière permet de comprendre que sa défense a priori inactuelle d'une rhétorique en voie de disparition (en tout cas institutionnelle) s'explique pleinement dès lors que l'on s'attache à la resituer dans le cadre d'une pensée et d'un parcours critique incessamment voués à la sauvegarde de la tradition et, plus exactement, à la promotion de tout ce qui confère à la littérature sa portée universelle. De même que ses éloges réitérés du classicisme ${ }^{8}$, l'apologie pour la rhétorique que nous présentons ici s'inscrit dans une réaction plus vaste contre le particularisme de la littérature moderne ${ }^{9}$ et poursuit les réflexions que Brunetière avait développées dans un texte antérieur, "Théorie du lieu commun », publié en $1881^{10}$. Le début de cet article, rédigé sous prétexte de rendre compte du Dictionnaire des lieuxcommuns de Lucien Rigaud ${ }^{11}$, montre que les batailles contre le cliché d'expression menées au début du xIX ${ }^{e}$ siècle par les Romantiques ont fait malgré tout leur chemin, y compris chez leur plus farouche adversaire. Brunetière concède en effet que, si on l'envisage comme un figement phraséologique historiquement déterminé, le lieu commun est sujet à l'usure et finit inévitablement par être anachronique, puisque sa pertinence et sa motivation dépendent des réalités de l'époque où il a fait son apparition $^{12}$. En revanche, dans une acception plus élargie, allant jusqu'à inclure la notion plus proprement rhétorique de topos $^{13}$, le lieu commun ne connait pas d'anachronisme : il s'avère au contraire pour Brunetière « la substance même de l'art de parler et d'écrire ${ }^{14}$ ", autrement dit une partie du fonds commun de la langue où tout le monde puise pour s'exprimer. Ne se privant pas des ressources du paradoxe, Brunetière va jusqu'à affirmer que les lieux communs sont «la condition même de l'invention en littérature ${ }^{15}$ », en ce qu'ils forment le réservoir des idées léguées par la tradition, à partir desquelles doit éclore toute création singulière. Ces idées sont inépuisables précisément parce que banales, et tout un chacun peut se les approprier selon ses propres exigences. Dès lors, le génie de l'artiste se manifeste non pas dans la 
poursuite à outrance de l'invention et du nouveau prônée par les romantiques, mais dans sa capacité à régénérer un contenu universel, en y apposant son cachet individuel.

Cette valeur patrimoniale du lieu commun est rappelée dans l'étude de 1890 , où Brunetière prend vigoureusement le parti de «l'illustre victime » qu'est désormais la rhétorique ${ }^{16}$. Afin de dissiper le flou sémantique qui caractérise ce terme, arboré tour à tour comme une bannière ou comme une "injure littéraire ${ }^{17}$ ", le critique revendique la conception humaniste selon laquelle la rhétorique constitue moins une collection de procédés artificiels et d'ornements qu'un art d'écrire, d'autant plus incontournable qu'il est consubstantiel à l'art de penser. Même si l'on en fait « dès que l'on écrit ${ }^{18}$ ", y compris quand on affecte de la mépriser, la rhétorique ainsi considérée n'est pas innée ; elle se compose au contraire de règles qui peuvent être enseignées ${ }^{19}$ et qui permettent d'agencer les idées de façon à ce qu'elles puissent être communiquées avec clarté. En ce sens, la rhétorique inclut aussi bien les principes et les instruments (à savoir les figures) qui élèvent la langue commune au rang d'œuvre $d^{\prime} \operatorname{art}^{20}$, que les prescriptions qui guident l'ordonnancement des parties et les transitions, autrement dit la dispositio, ou « l'art de composer ${ }^{21}$ ». Dans cette acception plus large, la rhétorique, comme déjà le lieu commun, s'avère pour Brunetière le gage de la mission sociale de la littérature en tant qu'élément fédérateur d'une communauté. Indissociable de l'activité de production d'une parole conçue par et pour les (honnêtes) hommes, elle en vient à représenter pour Brunetière une culture, un patrimoine universel qui, répondant à "quelque besoin général, intérieur et profond de la littérature et de l'humanité22", permet de dépasser l'individualisme ainsi que le dogme de singularisation qui régissent l'époque moderne (et les productions littéraires qui en émanent).

6 Une fois ces jalons définitoires posés, Brunetière précise le rayon d'action et le positionnement de la rhétorique par rapport à d'autres disciplines telles que la logique ou la dialectique. Son but n'est pas la démonstration mais la persuasion, et son domaine s'étend sur « toute une province de l'âme humaine ${ }^{23}$ » qui échappe à la juridiction d'une science par trop aride. Puisque pour Brunetière l'enjeu distinctif de la rhétorique est de rendre sensibles les vertus et les sentiments «qui font le prix de la société des hommes $^{24}$ ", il s'ensuit que - malgré l'importance accordée à la topique - l'elocutio et l'emploi des figures dans une visée pathétique occupent toujours le premier plan en ce qu'elles constituent les moyens pour transformer ces sentiments en «mobiles d'action ${ }^{25} »$. Dans cette optique, il n'y a pas lieu de considérer la rhétorique comme étant distincte de l'éloquence : à ceux qui condamnent volontiers la première en tant qu'arsenal de procédés mécaniques et superfétatoires ${ }^{26}$, Brunetière répond que le mauvais usage qu'on peut faire des ressources rhétoriques n'entache pas les mérites de la discipline elle-même. Elle seule, par ses préceptes et ses autorégulations, peut en effet fournir le remède contre les abus qui peuvent l'affaiblir; ce sont d'ailleurs les meilleurs rhéteurs - si honnis par les modernes pourfendeurs de la rhétorique - qui en ont dénoncé de tout temps les faiblesses et les artifices.

7 Les axes qui structurent l'argumentation de Brunetière dans «Apologie pour la rhétorique » montrent bien que, tout compte fait, l'ambition de son auteur n'est pas de redéfinir, sur de nouvelles bases, le statut ontologique de la discipline (sa conception se nourrit d'idées qui n'ont strictement rien de novateur), ni de contribuer à en repenser la place au sein de l'enseignement - et ce malgré son plaidoyer conclusif pour une coexistence de l'“ancienne" rhétorique à côté de la "nouvelle" histoire littéraire ${ }^{27}$. La rhétorique apparait bien davantage comme une arme supplémentaire à brandir dans 
un combat idéologique voué à «fortifier la tradition, à maintenir ses droits contre l'assaut tumultueux de la modernitée ${ }^{28}$ ». La mise en valeur de l'universalité et du caractère transhistorique des lieux et principes de la rhétorique constituent, de ce fait, la réponse défensive et conservatrice que Brunetière oppose aux tendances de plus en plus autotéliques de la littérature contemporaine, coupable d'avoir sacrifié à la tyrannie d'une "originalité décevante ${ }^{29}$ " les valeurs esthétiques et éthiques du classicisme, qui seul a réussi à conjuguer l'expression individuelle du génie et la portée universelle, proprement humaine, de l'art.

\section{Édition}

Initialement paru dans :

Revue des deux mondes, $3^{\mathrm{e}}$ période, t. 102, 1890 (livraison du $1^{\mathrm{er}}$ décembre),

p. 687-698 ; repris dans Essais sur la littérature contemporaine, Paris, Calmann-Lévy, 1892, p. 287-311. Nous suivons le texte de cette édition de 1892, en indiquant entre crochets droits les pages de sa réimpresssion de 1893 (sur Google Livres : https:// books.google.fr/books?

id=CytcAAAAcAAJ\&pg=PA292\&dq=inauthor:Bruneti\%C3\%A8re\&hl=fr\&sa=X\&ved=2ahUKEwjf4tT4n_DsAhWMsBQKHU5eB

8 S'il est, comme on l'a dit, des morts qu'il faut qu'on tue, n'en est-il pas peut-être aussi, de loin en loin, qu'il faut qu'on ressuscite, ou dont on essaye de ranimer et de renouveler la mémoire? C'est à quoi je songeais en lisant, tout récemment encore, l'invective d'un honnête homme de philosophe contre la rhétorique ${ }^{30}$, et je me demandais si le temps ne serait pas venu de plaider un peu la cause de cette illustre victime. Car enfin, s'il y a certainement une partie de l'art d'écrire, divine et comme inspirée, qui ne s'apprenne pas, qui ne se transmette point, inimitable et incommunicable, n'y en a-t-il point de plus humbles aussi, qui s'enseignent, et dont il y a vraiment des règles ou une théorie ? Personne, je pense, n'oserait prétendre qu'il n'y ait pas un art de chanter. C'est peu de posséder la plus belle voix du monde, et il faut encore savoir s'en servir, la diriger, la ménager. [288] Comment n'y aurait-il pas aussi un art de parler et d'écrire ? L'abus de la rhétorique en doit-il faire condamner l'usage ou méconnaitre l'utilité, je dirai tout à l'heure le prix ? Et parce que quelqu'un aura dit que « la vraie éloquence se moque de l'éloquence ${ }^{31}$ ", l'en croirons-nous sur sa parole? ou prétendrons-nous peut-être, avec un autre, qu'on écrit toujours assez bien quand on parvient à se faire entendre ${ }^{32}$ ? Je ne sache pas, en ce cas, de cuisinière ou de palefrenier qui n'y réussisse aussi bien qu'un académicien.

9 Ah! sans doute, si nous ne parlions jamais que pour agir; si nous étions toujours uniquement guidés, dans tout ce que nous écrivons, par des intérêts supérieurs à nousmêmes, des intérêts où celui de notre amour-propre ne fût jamais mêlé ; si nous ne nous proposions que d'instruire, de gagner ou de convertir des âmes; si nous étions Pascal, - puisque je viens de le citer, - ou Bossuet, ou Bourdaloue seulement, alors, oui, nous pourrions affecter ce mépris de la rhétorique ! Nous en pourrions rejeter loin de nous les "ornements", et les "artifices». Nous aurions le droit de dédaigner, pour notre parole comme pour notre personne, «tout ce que les hommes admirent ${ }^{33}$ ». Et cependant, et encore, Pascal lui-même, pourquoi donc récrivait-il jusqu'à sept ou huit fois chacune de ses Provinciales $^{34}$ ? Pourquoi Bossuet, plus désintéressé que [289] Pascal, 
refaisait-il, aussi lui, ses Sermons? Pourquoi revoyait-il si soigneusement le texte de ses Oraisons funèbres ou de son Histoire universelle? Pour en assurer la doctrine, je le sais; et je le veux bien ; mais aussi, tous les deux, pour que la force de leur parole fit entrer plus sûrement leurs idées dans les esprits de leurs lecteurs ou de leurs auditeurs. Ils avaient donc beau la mépriser, ils faisaient de la rhétorique! S'ils ne lui laissaient pas prendre, dans leurs discours, plus de place qu'elle n'en doit occuper, ils en usaient pourtant ! Ils savaient le "pouvoir d'un mot mis en sa place $^{35}$ "; ils connaissaient aussi celui d'une " cadence harmonieuse ${ }^{36}$ "! Ayant affaire avec des hommes, ils les prenaient par des moyens humains. Cela ne valait-il pas mieux que de les rebuter d'abord, et, voulant nous dire quelque chose, devaient-ils commencer par nous décourager ou par nous dégoûter de les entendre ? Mais ce qui est vrai de ces grands hommes, combien ne l'estil pas davantage de nous, je veux dire de tous les [290] écrivains qui ne sont ni des apôtres, ni des conducteurs d'âmes, qui écrivent « pour se faire plaisir » à eux-mêmes, peut-être, mais aussi pour que l'on les lise, comme le peintre pour qu'on le regarde, comme le musicien pour qu'on l'écoute! Je ne passe le mépris ou le dédain de la rhétorique qu'à ceux-là seuls qui n'impriment point, qui n'imprimeront jamais, qui ne laisseront pas non plus de Mémoires derrière eux, qui se garderont enfin toujours d'écrire ; - fût-ce contre la rhétorique, puisqu'on en fait dès que l'on écrit.

Il est vrai qu'il faudrait s'entendre sur ce mot même de rhétorique; et c'est ce qui n'est pas facile, depuis qu'on l'a détourné de son ancien sens, de celui qu'il avait encore au temps de Bossuet et de Pascal, pour en faire une espèce d'injure littéraire. Aussi bien vivons-nous dans un temps où chacun se plaît d'attacher aux mots le sens qui lui convient, sans se préoccuper autrement ni de leur signification, ni de leur histoire, ni de leur origine. Qu'est-ce que voulait dire, par exemple, M. Ernest Renan, dans la Préface du tome III de son Histoire du peuple d'Israël, quand, à ceux qui ne voient pas entre Félix Pyat et le prophète Jérémie la ressemblance dont il s'égaye lui-même, il reprochait assez aigrement « leur susceptibilité de rhéteurs ${ }^{37}$ »? Il ne se proposait, j'imagine, que de leur être désagréable; car quelle "rhétorique» peut-il y avoir à trouver une ressemblance douteuse, une [291] comparaison mauvaise, un rapprochement malheureux; - et à le dire, très simplement? On peut le dire, on peut avoir d'autres idées que M. Renan sur les prophètes, et n'être pas un "rhéteur " pour cela! Mais, à son tour, M. Maxime Du Camp, dans son Théophile Gautier, lorsqu'il nous dit qu'avec les vers de Musset, ceux de Gautier sont les seuls de leur temps qui ne soient pas " entachés de rhétorique ${ }^{38}$ ", comment l'entend-il ? Et nous, comment l'entendronsnous? Car j'aurais pensé jusqu'ici qu'il n'y avait point de "rhétorique », ou bien peu, dans Jocelyn ${ }^{39}$ et dans les Destinées ${ }^{40}$, par exemple, dans les vers de Lamartine et dans ceux de Vigny ; mais j'en trouvais beaucoup, au contraire, et bien plus que je n'en eusse voulu, dans Albertus ${ }^{41}$ et dans Rolla ${ }^{42}$.

Regrettez-vous le temps où le ciel sur la terre,

Marchait et respirait dans un peuple de dieux...

Dors-tu content, Voltaire, et ton hideux sourire

Voltige-t-il encor sur tes os décharnés...

Cloîtres silencieux, voûtes des monastères,

C'est vous, sombres caveaux, vous qui savez aimer ${ }^{43}$...

Qui jamais a plus abusé que Musset de l'exclamation, et de l'apostrophe, et généralement de tout ce qu'il y a de "figures" cataloguées dans les traités des rhéteurs? Mais, pour Gautier, n'est-il pas plaisant qu'on veuille exempter aujourd'hui $\mathrm{du}$ reproche de rhétorique celui de tous nos contemporains qui a [292] cru le plus fermement au pouvoir des « vocables ", à leur valeur propre et intrinsèque, extérieure 
et supérieure à celle des idées qu'ils expriment? Pour ne pas nous égarer parmi toutes ces contrariétés, tenons-nous-en donc aux anciennes définitions, et prenons ici le mot comme on l'a toujours pris, depuis Aristote jusqu'à Fénelon. La rhétorique est l'ensemble des règles et des lois qui gouvernent l'art d'écrire, considéré lui-même comme inséparable de l'art de penser; et, qu'on le sache ou non, - mais je crains qu'on ne le sache fort bien, - ce que l'on nie quand on attaque la rhétorique, c'est qu'il y ait un art de penser et d'écrire.

11 En quoi consiste-t-il ? Je me garderai bien de le vouloir préciser. On ne manquerait pas de me demander si je crois donc le posséder moi-même ; et il est vrai que la plaisanterie ne signifierait rien ; mais j'aime mieux ne procurer à personne une trop belle occasion de la faire. Les règles ou les lois en sont d'ailleurs dans toutes les Rhétoriques; et Quintilien ou Aristote disent là-dessus de fort bonnes choses, qui le sont pour nous comme pour les Grecs ou les Latins. Mais ce qui sera plus intéressant peut-être, ce sera de rappeler quels sont les principes de cet art, ou encore les raisons, les éternelles et solides raisons qu'il y aura toujours d'en faire cas. Non seulement ce n'est rien d'aussi futile et d'aussi puéril qu'on le dit quelquefois, que [293] d'apprendre à écrire, mais il se pourrait que ce fût quelque chose d'essentiel. Née de bonne heure, on le sait, et presque contemporaine des origines de la littérature grecque, la rhétorique doit sans doute répondre, et je crois qu'en effet elle répond à quelque besoin général, intérieur et profond, de la littérature et de l'humanité.

12 «C'est trop peu estimer le public de ne prendre pas la peine de se préparer quand on traite avec lui. Et un homme qui paraitrait en bonnet de nuit et en robe de chambre, un jour de cérémonie, ne ferait pas une plus grande incivilité que celui qui expose à la lumière du monde des choses qui ne sont bonnes que dans le particulier, et quand on ne parle qu'à ses familiers ou à ses valets ${ }^{44}$. Ainsi s'exprime quelque part Balzac, l'autre Balzac - celui des deux que Sainte-Beuve préférait, pour des raisons toutes personnelles - et dont il a si bien dit qu'il avait précisément fait faire sa « rhétorique » à la prose française ${ }^{45}$. Combien de gens qui n'écriraient pas, si l'on exigeait, si l'on pouvait exiger d'eux qu'avant d'écrire ils eussent médité cette leçon de l'ancienne politesse ! Combien de Mémoires, et de Journaux, et de Confessions, dont la littérature serait heureusement privée, si nous savions nous-mêmes distinguer entre ce qui n'est bon que pour nos « familiers ", ou nos " valets », - quand nous en avons, - et ce qui vaut la peine qu'on l'expose «à la lumière du monde »! Voilà le premier [294] principe de toute rhétorique. C'est pour soi, mais c'est aussi pour les autres qu'on écrit et qu'on parle ; et, assurément, nous ne devons ni leur sacrifier, ni déguiser pour eux ce que nous croyons être la justice et la vérité, mais nous devons les leur présenter d'une manière qui ne heurte pas trop rudement leurs oreilles, leurs habitudes, et leurs préjugés. N'est-ce pas ainsi, - je crois que la remarque vaut bien qu'on la fasse en passant, - n'est-ce pas ainsi qu'a grandi, que s'est développée notre littérature classique? Je ne parle plus de Balzac maintenant. Mais soyez sûr que l'auteur des Provinciales, s'il n'avait pas pris le soin de mettre d'abord « le monde » de son côté, n'eût jamais réussi à insinuer dans les esprits de son temps quelque chose de la sévérité de la morale janséniste. Et, en vérité, les moyens qu'il en a choisis, c'était de l'excellente rhétorique, mais c'était de la rhétorique...

13 Souvenons-nous, en effet, ici, que la littérature, comme l'art en général, a vraiment une fonction, et je serais tenté de dire une mission sociale. C'est le sens profond des anciens mythes qui plaçaient l'éloquence à l'origine des civilisations ou des sociétés mêmes. $\mathrm{Ne}$ 
savons-nous pas bien, d'ailleurs, que, si les grands peuples prennent quelque part une pleine conscience de ce qu'ils sont, c'est dans leur littérature ? Et, divisés que nous sommes de toutes les manières, par nos intérêts ou par nos [295] passions, n'est-ce pas la littérature encore qui rétablit tous les jours une solidarité qu'au contraire l'attrait des plaisirs égoïstes et l'âpreté de la lutte pour la vie tendent perpétuellement à dissoudre ? Une ode ou une élégie, un drame ou un roman n'opèrent sur le lecteur, si je puis ainsi parler, qu'autant qu'ils éveillent ou qu'ils font naître en lui des "états d'âme" voisins de celui du romancier, de l'auteur dramatique, ou du poète. La connaissance de ces "états d'âme ", en ce qu'ils ont de plus général et de plus humain, et par conséquent, l'art ou la science des moyens de la provoquer, c'est ce que les anciens rhéteurs appelaient «la topique». Changeons le mot, si peut-être nous le trouvons aujourd'hui trop grec, trop pédantesque, trop rébarbatif: la chose n'en demeure pas moins. Un peu de topique eût jadis empêché Corneille d'écrire sa Théodore, son Pertharite, son Attila. Elle pourrait empêcher nos romanciers contemporains de prendre des états plus que particuliers, exceptionnels et morbides, pour des états ordinaires et généraux de l'âme humaine. Tout au moins, en les décrivant, sauraient-ils peut-être alors les rattacher à ces états moins exceptionnels dont ils ne sont qu'une aberration. Enfin, comme autrefois, chacun de nous abonderait sans doute moins dans son sens individuel, et je ne sais ce qu'il adviendrait de la littérature, mais, en se mêlant davantage à la vie de tout le monde, elle se rapprocherait assurément de [296] son véritable objet. On ne croirait pas que l'originalité consiste à ne ressembler à personne, mais uniquement à faire passer dans ce que l'on écrit son expérience personnelle du monde et de la vie. Et ce serait toujours de la rhétorique, mais j'ose dire que c'en serait encore de la bonne et de l'excellente.

Voici qui va plus loin, peut-être. Si vous y regardez d'assez près, vous verrez qu'au fond, ce que l'on attaque sous le nom de rhétorique, c'est tout ce qu'il y a de moyens pour persuader aux hommes les choses qui ne se démontrent point. On ne démontre ni la liberté, ni l'immortalité, ni même la morale: on les persuade. On ne prouve pas la nécessité d'obéir, ni celle de vaincre ses passions, ni celle de se sacrifier : mais on y incline les cœurs. C'est ce que ne peuvent tolérer ceux qui, pour eux, ne veulent croire, comme ils disent, qu'à ce qui se prouve. Aussi, sous le nom de rhétorique, - avec un dédain qui ne va pas sans quelque colère, - enveloppent-ils indifféremment tout ce qu'ils craignent qui gêne ou qui contrarie leurs propres convictions. Rhétorique, une Provinciale de Pascal! Rhétorique, un sermon de Bossuet, sur l'Honneur du monde ou sur la Haine des hommes contre la véritét ! Rhétorique, un Discours de Rousseau, son Contrat social ou sa Profession de foi du vicaire savoyard ${ }^{47}$ ! Rhétorique, le Génie du christianisme ${ }^{48}$ ou l'Essai sur l'indifférence ${ }^{49}$ ! Et, généralement, [297] rhétorique, tout ce qu'ils sentent bien qui va, non pas du tout contre la vérité, - puisqu'elle nous échappe, hélas! en toutes ces matières, - mais contre les idées ou contre les principes dont ils ont décidé, qu'à défaut d'elle, et parce qu'il faut bien vivre, ils s'accommoderaient. Je n'ai pas besoin, quant à moi, d'un plus bel éloge de la rhétorique ; et plus j'y ai songé, plus il m'a semblé qu'en même temps que la raison cachée des attaques si vives qu'on dirige contre elle, c'était là précisément son fort.

Oui ; - là où viennent expirer le pouvoir de la logique et celui de la dialectique, là commence le pouvoir de la rhétorique. Où le raisonnement s'égare, et où la raison même gauchit, c'est là qu'elle intervient et qu'elle fonde son empire. Toute une province de l'âme humaine, et non pas la moins vaste, inaccessible, impénétrable aux démonstrations des savants et aux inductions de la métaphysique, elle s'en empare, elle 
s'y établit, et elle y règne souverainement. « Dites-moi, demandait Cicéron, - tout au début de l'un de ses traités de rhétorique, parmi lesquels il y en a qui valent ses discours, - dites-moi comment les hommes auraient jamais pu se plier à l'observation de la probité et de la justice ; comment ils auraient consenti à incliner leur volonté sous celle de l'un de leurs semblables; comment on leur aurait persuadé de faire cause commune dans l'intérêt commun; et, à [298] cet intérêt, de sacrifier au besoin leur vie même, si ce n'était à l'aide et par le moyen de la persuasion, de l'éloquence et de la rhétorique $^{50}$ ?» Et, en effet, probité, charité, justice, vertu, amour de la patrie, tout ce qu'il y a de sentiments qui font le prix de la société des hommes, et que non seulement l'instinct, toujours égoïste, mais que la raison même, toujours calculatrice, nous déconseille, c'est elle, c'est l'éloquence et c'est la rhétorique qui les rendent sensibles aux cœurs, qui leur prêtent une voix et des gestes, qui les font parler, si je puis ainsi dire, aux corps mêmes. Telle est l'origine de leurs «figures »; tel aussi l'objet de leurs " mouvements »; telle est l'explication de leur puissance. En matérialisant ce qui ne se voit ni ne se touche, la rhétorique en fait des motifs actuels, ou, pour mieux dire encore, des mobiles d'action. Les «rhéteurs » du XvI siècle ont fait la réforme, et les « rhéteurs » du XVIII ${ }^{\mathrm{e}}$ ont fait la révolution, qui sont peut-être d'assez grandes choses ; quoi que d'ailleurs on en puisse penser. C'est qu'ils ont agi, comme rhéteurs, au lieu où se prennent les grandes résolutions, et c'est que leur pouvoir était comme inhérent à ce qu'il y a de plus profond dans la nature humaine. Nous ne vivons pas seulement de pain, d'algèbre et d'exégèse, mais de toute parole qui vient du cœur de nos semblables et qui pénètre jusqu'au nôtre. Si la rhétorique est l'art de faire valoir cette parole, - et c'en est une définition que je ne crois pas [299] qu'on puisse me disputer, - ni la logique ni la dialectique ne prévaudront jamais contre elle; et, bien loin de s'en plaindre, il me semble qu'il convient plutôt que l'on s'en félicite.

16 Car il n'importe pas qu'on en puisse faire un mauvais usage. De quoi ne peut-on mésuser? Corruptio optimi pessima est ${ }^{51}$. Si la rhétorique avait moins de pouvoir pour le bien, elle en aurait moins aussi pour le mal ; et puis la science, qu'on lui oppose, est-elle donc si sûre de n'avoir jamais fait que du bien? On montrerait aisément qu'elle aurait tort de le croire; et, plus d'un service que nous devons aux savants, l'humanité l'a chèrement payé. Mais ce qui est encore plus certain, c'est qu'une démonstration n'a jamais triomphé d'un sentiment, et que, par suite, s'il y a une mauvaise rhétorique, tout ce que nous pouvons contre elle, c'est de lui en opposer une meilleure. On ne répond, si je puis ainsi dire, à un Discours que par un Discours, on ne répond à un Sermon que par un autre Sermon, - Démosthène contre Eschine, Bossuet contre Calvin, - et, pourquoi n'irais-je pas jusque-là ? on ne répond à une prosopopée que par une hypotypose, et à une métonymie que par une synecdoque. Ou, en d'autres termes encore, on ne substitue point dans les cœurs « la vérité » à «l'erreur ", mais une croyance à une autre croyance, un sentiment à un autre, une volonté plus ferme à une volonté plus molle, et un motif d'agir plus persuasif à un mobile [300] d'action plus lent et plus paresseux. De telle sorte que, proscrire la rhétorique sous le prétexte des maux qu'elle a causés et de l'abus qu'on peut faire de ses exemples ou de ses leçons, je crois, et peut-être voit-on, que ce serait tout simplement se désarmer contre elle. Nous avons besoin d'elle contre elle-même. Puisqu'elle répond à une nécessité de la nature humaine, il faut qu'on s'y résigne ; et, si je me suis clairement expliqué, cette nécessité, c'est la plus impérieuse de toutes, - plus impérieuse, assurément, que le besoin de savoir ou de voir, - puisque c'est la nécessité d'agir. 
17 On dira, je le sais, que je confonds ici la rhétorique avec l'éloquence? J'aimerais alors qu'on voulût bien aussi me dire où est la différence. Car, fût-il Démosthène, Cicéron ou Bossuet, je ne sache guère d'orateur que l'on n'ait accusé de déclamation; et j'ai même observé qu'en général il suffisait, pour cela, qu'on ne pensât pas comme lui. Bossuet, par exemple, qui est un rhéteur pour Voltaire, quand il écrit son Discours sur l'Histoire universelle ${ }^{52}$, n'en est pas un pour l'auteur des Soirées de Saint-Pétersbourg ${ }^{53}$; mais qu'il prononce le Sermon sur l'unité de l'Église ${ }^{54}$, c'est assez, et il en redevient un pour l'auteur du Pape et de l'Église gallicane ${ }^{55}$. La vérité, la voici donc : c'est que, d'orateur à rhéteur, il n'y a de différence que celle de la solidité des choses qu'ils disent; et, comme cette solidité n'est et ne peut être jamais fondée que dans l'opinion de ceux [301] qui les écoutent, la différence, on le voit, n'est pas grande. Si cependant, prenant le mot de rhétorique dans son sens le plus étroit, et laissant là le fond des choses pour ne nous attacher uniquement qu'à la forme, nous acceptons la définition qu'en donnent ceux-là mêmes qui la méprisent le plus, les arguments ne manquent pas encore pour leur répondre, nombreux et décisifs, parmi lesquels je n'en choisirai qu'un.

Une langue est-elle un organisme ? On le dit ; je n'en sais rien, et je ne le crois pas ; mais ce qu'elle est certainement, ce qu'elle devient dès qu'on s'en sert pour autre chose que pour les besoins de la vie quotidienne, c'est une œuvre d'art. Die Sprache als Kunst ${ }^{56}$ : ce titre d'un livre allemand me plait. Ce que les couleurs et les lignes sont en effet dans les arts plastiques, ou les sons encore en musique, les mots le sont dans une langue, et, à plus forte raison, les «figures », les tours, la disposition des parties de la phrase. Il y a de beaux mots, qui sonnent bien à l'oreille, il y en a d'odieux, qui l'offensent, qui la blessent, qui remplissent aussi l'imagination d'idées communes, vulgaires, ou impures. Mais que dis-je, des mots? c'est des syllabes qu'il faut dire, c'est une simple combinaison de consonnes et de voyelles. Vous en trouverez dans nos Dictionnaires d'argot autant d'exemples que vous en voudrez. Osera-t-on soutenir que l'art soit méprisable ou seulement indifférent, qui s'efforce d'éviter [302] ces rencontres ou ces concours de sons, ces mots de prison ou bagne, et s'il ne peut pas toujours absolument les éviter, qui fait du moins son possible pour les dissimuler? Mais si, selon le mot de Pascal, « le seul ton de la voix change un poème ou un discours de face ${ }^{57}$ ", l'accent, le tour, le mouvement ne suffisent-ils pas à modifier le sens d'une phrase? Rien que de renverser l'ordre des mots d'une phrase, on la rend claire d'obscure qu'elle était; vive et légère celle qui était lourde; nombreuse et harmonieuse, de rude et de cacophonique. Et les métaphores, qui longtemps avant d'être des «ornements du discours ", ont été, sont encore le moyen ou le procédé naturel de développement et, pour ainsi parler, de fructification des langues? C'est l'imagination qui les trouve, mais si la rhétorique est l'art de s'en servir, de ne pas confondre une antithèse avec une similitude, si surtout elle nous apprend quand et comment on en use, avec quelle mesure, et pour l'expression de quelles idées ou de quels sentiments, qui ne voit que, même ainsi prise, comme je disais, dans son sens le plus étroit, la rhétorique mène toujours et nécessairement de l'art d'écrire à celui de penser?

19 J'aurais vraiment la partie trop belle, si je voulais montrer qu'elle est encore l'art de composer. Mettre de l'ordre dans ses pensées, mesurer à leur importance le développement que l'on en donne, passer de l'une à l'autre par des transitions qui ne [303] s'aperçoivent point, régler l'allure de son mouvement sur quelque chose de moins capricieux que notre humeur, - je dirais presque de moins capricant, - c'est ce que de fort grands écrivains n'ont point su, faute d'un peu de rhétorique, un Montesquieu par 
exemple, et un Chateaubriand. En sont-ils moins grands pour cela? demandera-t-on peut-être. Non ; mais ils n'en sont pas plus grands, je pense ; et l'Esprit des lois ou le Génie $d u$ christianisme, moins bien composés, en sont par cela même, l'un moins clair, moins intelligible, et l'autre, le second, moins persuasif ou moins démonstratif. Si, d'ailleurs, nul ne peut se flatter lui-même d'être Chateaubriand ou Montesquieu, c'est sans doute une raison de leur laisser leurs défauts, qui ne sauraient être couverts ou excusés que par des qualités égales ou analogues aux leurs. En attendant, on ne court aucun risque, s'il existe un art de composer, et qu'il s'enseigne, de l'apprendre. Notez encore que ce genre de règles contient en soi le moyen même de s'en passer, s'il y a lieu. Savoir ce qu'il ne faut pas faire, c'est une partie de la justice, et une partie assez étendue, puisqu'en tout pays nous voyons que les codes roulent sur elle. La rhétorique nous apprend pareillement ce qu'il ne faut ni écrire ni dire. Mais elle nous apprend de plus ce qu'il faut faire, et il ne s'ensuit pas que nous puissions le faire, mais en vérité, je ne vois pas ce qu'il peut y avoir de mal à tenter de le faire.

[304] Rappelons-nous enfin que ce sont ces rhéteurs qu'on méprise ou dont on se moque, ces peseurs jurés de mots et de syllabes, ces " greffiers » de l'usage, ces curieux en l'art de bien dire, ces éducateurs de la mode, un Balzac, un Vaugelas, les précieuses elles-mêmes, La Bruyère, Fénelon aussi, Voltaire surtout, un Rollin, un Rivarol, combien d'autres encore ? - ce sont eux qui ont fait de notre prose française le souple et le flexible à la fois, le délicat et le pénétrant, l'admirable instrument qu'elle est ; - ou qu'elle fut. Cette rhétorique plus haute qu'on trouve, quand on l'y cherche, dans les écrits d'un Chateaubriand ou d'un Rousseau, d'un Bossuet ou d'un Pascal, ils l'en ont dégagée, et ils l'ont mise à notre portée. On ne savait pas ce que c'était que le style naturel : Pascal a paru qui nous l'a révélé, et on en a senti aussitôt tout le prix. Mais en quoi ce style naturel consiste, et s'il y en a quelques secrets que l'on puisse dérober à l'auteur des Provinciales, ce sont les rhéteurs qui l'ont cherché, qui nous en ont indiqué les moyens, et qui ont enrichi la langue, si je puis ainsi dire, des «propres» de Pascal. Inversement, dans un autre écrivain, l'auteur du Petit Carême ${ }^{58}$, par exemple, s'il y a trop de vains ornements, trop de désir de plaire, trop de jolies choses, et généralement plus de souci de lui-même que de son sujet, - ce qui pourrait bien être la définition même de la mauvaise rhétorique, - ce sont [305] encore les rhéteurs qui nous l'ont dénoncé, qui nous en ont dévoilé l'artifice, qui nous ont fait sentir l'abus de la rhétorique dans l'usage de ces procédés mêmes. Je ne puis croire qu'ils nous aient rendu là de si mauvais services; et qui continuerait leur tâche parmi nous, je n'estimerais pas qu'il y perdît son temps.

Quelques-uns s'en sont avisés, dont on ne se doute pas, parce que nous ne savons plus reconnaître, sous la diversité des mots, la ressemblance des choses. Étant entendu que la rhétorique est un legs du passé - ce qui suffit, auprès de bien des gens, pour la discréditer - nous ne faisons aucun cas des rhéteurs, mais nous en faisons un tout particulier des stylistes. Est-ce que pourtant Gautier ne faisait pas de la rhétorique, - et de la bien mauvaise, pour le dire en passant, - quand il écrivait son Capitaine Fracasse? Est-ce qu'il n'en tenait pas ouvertement école quand il répétait un de ses mots favoris : "Je suis très fort, j'amène cinq cents au dynamomètre, et je fais des métaphores qui se suivent ${ }^{59}$. On a même si bien retenu le conseil, qu'ouvrez vos journaux, et vous verrez que l'unique mesure qu'il y ait de la valeur du style d'un écrivain, ce n'est pas même la justesse, mais la « cohérence » de ses métaphores. Une métaphore incohérente ! qu'on le renvoie à l'école! Et on ne fait pas attention que l'un des principaux caractères de l'affectation et de la préciosité du style, c'est précisément la [306] «cohérence » des 
métaphores ${ }^{60}$. Mais la Correspondance tout récemment publiée de Flaubert, qu'est-elle, en vérité, qu'un cours de rhétorique, où j'avoue d'ailleurs très volontiers qu'on trouve d'excellentes leçons? En voici une qu'il me paraît piquant de relever au passage: «Nous nous étonnons des bonshommes du siècle de Louis XIV, mais ils n'étaient pas des hommes d'énorme génie, - et j'en connais au moins quatre sur lesquels il se trompe, - mais quelle conscience! Comme ils se sont efforcés de trouver pour leurs pensées les expressions justes! Quel travail! Comme ils se consultaient les uns les autres! Comme ils savaient le latin! Comme ils lisaient lentement! Aussi toute leur idée y est; la forme est pleine, bourrée et garnie de choses jusqu'à la faire craquer ${ }^{61}$. Est-ce ou non de la rhétorique? et je ne dis pas de la plus fine, - il n'y a guère de mot qui convînt moins à Flaubert, - mais de la bonne, et presque de la meilleure?

Que si, cependant, ces considérations, un peu sommaires, ne réussissaient pas à désarmer et à toucher [307] quelques dédaigneux, on leur en pourrait offrir de plus utilitaires, - et de fort érudites en même temps. On leur demanderait pourquoi les Latins et les Grecs ont si passionnément cultivé la rhétorique. Et je ne vois pas ce qu'ils pourraient répondre, sinon que, dans les républiques de l'antiquité, la parole étant une arme, quiconque voulait agir, il fallait bien qu'il en connût le maniement ou l'escrime? Dans Athènes comme dans Rome, qui n'eût point su parler n'eût pas pu seulement se défendre, et il fallait qu'il fût à peu près immanquablement de la clientèle ou de la domesticité politique d'un plus éloquent. Lisez là-dessus Fénelon, dans sa Lettre à l'Académi $^{62}$. Nous donc, qui vivons aujourd'hui sous le gouvernement de la parole, dont on peut dire que nos intérêts quotidiens sont à la merci d'un discours ou de l'impossibilité d'y répondre, il nous faut apprendre à parler, et, comme les Grecs ou les Romains, nous avons de la rhétorique plus de besoin que n'en avaient nos pères. Nous en avons besoin, quand ce ne serait que pour rétorquer ou, comme on disait jadis, pour reboucher celle de nos adversaires... Mais si j'insistais sur cet argument, je craindrais de mêler, dans une question jusqu'ici toute littéraire, des raisons qui le seraient moins et que, pour ce motif, il me suffit d'avoir indiquées... Après tout, les grands ennemis de la rhétorique sont peut-être ceux aussi du gouvernement de la parole : la liberté qu'ils aiment est à la muette ${ }^{63}$; et le droit [308] qu'ils revendiquent énergiquement pour les autres, c'est celui de se taire.

Une autre raison me paraît d'ailleurs plus forte, et c'est par là que je veux terminer. On a rayé la rhétorique, voilà déjà quelques années, du programme de notre enseignement secondaire, pour l'y remplacer par de vagues « notions d'histoire littéraire »; et, si j'ose prendre une fois la liberté de parler en mon nom, ce n'est pas moi qui me plaindrai qu'on ait fait quelque chose pour l'histoire littéraire. Il est bon que l'on sache, au sortir du collège, que le vieux Corneille, par exemple, en son Cinna, n'a point voulu flatter Louis XIV sous le nom d'Auguste. On l'ignorait, il n'y a pas longtemps. La rhétorique est une bonne chose, la chronologie en est une autre, et dois-je le dire ? elle fait une de mes passions. Mais, puisqu'on parle aujourd'hui beaucoup de la constitution future d'un "enseignement classique français », il ne paraîtra pas inutile d'exprimer le vœu que la rhétorique y reprenne sa place naturelle, et l'on ne trouvera pas mauvais que j'en donne le principal motif. C'est que notre littérature classique, - et je ne dis pas seulement la prose, je dis aussi la poésie, - est essentiellement oratoire. « La parole qui se prononce, disait Vaugelas dans la Préface de ses Remarques sur la Langue française, est la première en ordre et en dignité, puisque celle qui est écrite n'est que son image, comme l'autre est l'image de la pensée ${ }^{64} »$; et de Malherbe jusqu'à Buffon au [309] 
moins, jusqu'à Chateaubriand, jusqu'à Guizot, je ne vois guère que quelques conteurs dont la manière d'écrire ne vérifie pas le principe. Encore sait-on le cas que faisait de l'harmonie de la phrase l'auteur de Madame Bovary. Qu'est-ce à dire, sinon que, pendant deux ou trois cents ans, nos plus grands écrivains se sont non pas vus, mais qu'ils se sont entendus écrire. Voulez-vous voir s'évanouir la plupart des chicanes qu'on fait au style de Molière : ne vous contentez pas de le parcourir des yeux, allez le voir jouer, ou lisez-le vous-même à haute voix. Or comment, sans un peu de rhétorique, pourrait-on interpréter une telle littérature ? et ne perdrait-on pas la moitié du profit que l'on croit en tirer? Ce serait oublier, comme on dit, d'allumer sa lanterne. Essayez donc d'expliquer Racine, son Andromaque ou son Britannicus, sans insister sur cette ironie qui est un des moyens qu'il aime de nuancer sa pensée, et dont on peut dire qu'il semble avoir voulu épuiser tous les tours! Ou bien encore essayez de faire sentir ce que les Sermons de Bossuet ont d'unique sans essayer de faire toucher du doigt ce qu'ils ont de supérieur à ceux de Bourdaloue, et réussissez-y sans le secours de la rhétorique! Sans la rhétorique on peut être assuré que « l'enseignement classique français » dégénérera promptement en un "enseignement de choses ", et ce n'est pas sans doute ce que l'on veut, - ou du moins ce que l'on nous promet. Cette raison seule aurait suffi [310] pour m'engager à prendre la défense de cette dédaignée. J'espère cependant que le lecteur approuvera les autres, et qu'en les joignant toutes ensemble il voudra bien convenir avec nous qu'il est décidément des morts qu'il faut parfois qu'on ressuscite.

$$
1^{\text {er }} \text { décembre } 1890 .
$$

\section{NOTES}

1. En cette même année, Brunetière est élu à l'Académie française, où il succède au journaliste John Lemoinne. Les années 1893-1894 marquent la consécration de Brunetière, dont les débuts dans le milieu universitaire furent difficiles, en tant qu'intellectuel et vedette, écoutée ou honnie, dans le débat public. Pour un résumé détaillé de la biographie de Brunetière, voir A. Compagnon, Connaissez-vous Brunetière?, Paris, Seuil, 1997, p. 13-28.

2. F. Brunetière, "Le Symbolisme contemporain", Essais sur la littérature contemporaine, Paris, Calmann-Lévy, 1892, p. 135 : « Pornographie et reportage, voilà tout ce que l'auteur de L'Argent a su faire d'une grande doctrine d'art [...] Ce laborieux et puissant ouvrier de lettres, moins laborieux que régulier peut-être, et assurément moins puissant que commun, n'a pas compris ni ne comprendra jamais qu'en rendant le mot de naturalisme synonyme de celui de grossièreté, son œuvre manquait à toutes les promesses du nom qu'elle avait usurpé.» Voir aussi id., Le Roman naturaliste, Paris, Calmann-Lévy, 1883.

3. F. Brunetière, «Les artistes littéraires", Essais sur la littérature contemporaine, op. cit., p. 218 : « En même temps qu'un mystificateur, Baudelaire fut un malade et peut-être le commencement d'un fou ». Voir aussi id., "Charles Baudelaire », Questions de critique, Paris, Calmann-Lévy, 1889.

4. Voir F. Brunetière, «Symbolistes et décadents ", Nouvelles questions de critique, Paris, Calmann Lévy, 1890 ; "Le symbolisme contemporain », op.cit. Sur les rapports entre Brunetière et les jeunes littérateurs, nous renvoyons à D. Pernot, "Brunetière et la "jeune littérature" : tradition et canon », Revue d'Histoire littéraire de la France, n 114, 2014/1, p. 99-109. 
5. F. Brunetière, «L'organisation de l'enseignement secondaire français ", Revue des Deux Mondes, 1891, repris dans Essais sur la littérature contemporaine, op. cit., p. 311-336.

6. Propos de Louis Liard, directeur de l'enseignement supérieur entre 1884 et 1902, cités par A. Compagnon, "La Rhétorique à la fin du XIX e siècle", dans M. Fumaroli dir., Histoire de la rhétorique dans l'Europe moderne, Paris, PUF, 1999, p. 1129.

7. Voir aussi F. Brunetière, «L'enseignement de la littérature française dans la Faculté des Lettres », Revue internationale de l'enseignement, t. 1, janvier-juin 1881, p. 554-555.

8. Voir notamment «Classiques et romantiques », Revue des Deux Mondes, janvier 1883, repris dans Études critiques sur l'histoire de la littérature française, $3^{\mathrm{e}}$ série, Paris, Hachette, 1907, p. 291-326.

9. C'est-à-dire une littérature qui, d'une part, aborde des sujets trop particuliers, trop subjectifs et qui, d'autre part, fait un usage trop singulier (et donc obscur) de la langue : voir F. Brunetière, «Le Symbolisme contemporain », op. cit., p. 149-152.

10. F. Brunetière, «Théorie du lieu commun », Revue des Deux Mondes, 1881, repris dans Histoire et littérature, t. 1, Paris, Calmann-Lévy, 1884, p. 31-55.

11. Ce dictionnaire, paru la même année chez Ollendorff, rassemble un florilège des expressions clichéiques les plus fréquentes sous la plume des journalistes et des politiciens; de par son intention parodique, il participe de la haine contre le cliché caractéristique de la fin du siècle.

12. F. Brunetière, "Théorie du lieu commun ", Revue des Deux Mondes, op. cit., p. 453 : "Ainsi, ce qui fait qu'un lieu commun donne à rire, ce n'est pas qu'il est un lieu commun, c'est qu'il ne vient pas en son temps, c'est qu'il ne porte pas la marque du jour ».

13. Même si, dans la suite de son développement, on comprend que Brunetière entend par lieu commun moins le topos au sens étroit d'argument structurant une preuve que l'embryon de matière, le sujet minimal à partir de laquelle l'on crée une œuvre.

14. Ibid., p. 454.

15. Ibid., p. 455.

16. F. Brunetière, «Apologie pour la rhétorique », Revue des Deux Mondes, 1890, p. 687-698, repris dans Essais sur la littérature contemporaine, op. cit., p. 287-311.

17. Ibid., p. 290.

18. Ibid., p. 290.

19. Brunetière ne va pas pourtant jusqu'à prôner un enseignement fondé sur l'imitation des maîtres, comme le fera dix ans plus tard Antoine Albalat. Voir A. Albalat, La Formation du style par l'assimilation des auteurs, Paris, Armand Colin, 1901.

20. Ou de « belle prose ", selon les termes de G. Lanson dans L'Art de la prose [1908], Paris, La Table Ronde, 1996.

21. F. Brunetière, «Apologie pour la rhétorique », op. cit., p. 302.

22. Ibid., p. 293.

23. Ibid., p. 297.

24. Ibid., p. 298.

25. Ibid., p. 298.

26. C'était d'ailleurs déjà le reproche du philosophe John Locke (dans Essai sur l'entendement humain, [1689], trad. 1729, III, 10, 34) : «tout l'art de la Rhétorique, toutes ces applications artificielles et figurées qu'on fait des mots, suivant les règles que l'Éloquence a inventées, ne servent à autre chose qu'à insinuer de fausses idées dans l'esprit, qu'à émouvoir les Passions et à séduire par-là le jugement; de sorte que ce sont en effet de parfaites supercheries ».

27. La rhétorique est d'autant plus nécessaire que la littérature classique, qui doit constituer selon Brunetière le socle des programmes d'enseignement, est une littérature "oratoire » («Apologie pour la rhétorique», op. cit., p. 698), conçue moins pour être lue que pour être entendue, soit une littérature-discours, pour reprendre la formule d'Alain Vaillant («Pour une histoire de la communication littéraire ", Revue d'Histoire littéraire de la France, 3, 2003, p. 549-562). 
28. F. Brunetière, «Discours de réception à l'Académie Française ", repris dans Nouveaux essais de littérature contemporaine, Paris, Calmann-Lévy, 1897, p. 297-321.

29. Ibid., p. 319.

30. Nous n'avons pas pu élucider avec certitude quel «philosophe » se cacherait derrière cette trop vague allusion de Brunetière.

31. Pascal, Pensées, pensée $n^{\circ} 467$ [Sellier $n^{\circ}$ 671], série XXII, CEuvres complètes, II, éd. M. Le Guern, Paris, Gallimard, «Bibliothèque de la Pléiade », 2000, p. $744:$ «La vraie éloquence se moque de l'éloquence, la vraie morale se moque de la morale; c'est-à-dire que la morale du jugement se moque de la morale de l'esprit qui est sans règles. »

32. J.-J. Rousseau, «Lettre à M. du Peyrou », 12 avril 1765, Correspondance originale et inédite de J.J. Rousseau avec Mme Latour de Franqueville et M. Du Peyrou, II, Paris, Giguet et Michaud, 1803, p. 144: «Qu'une expression soit ou ne soit pas ce qu'on appelle française, ou du bel usage, ce n'est pas de cela qu'il s'agit : on ne parle et on n'écrit que pour se faire entendre ; pour qu'on soit intelligible, on va à son but; quand on est clair, on y va encore mieux : parlez donc clairement pour quiconque entend le français. "

33. J. B. Bossuet, "Panégyrique de l'Apôtre Saint-Paul ", CEuvres, textes établis et annotés par l'Abbé Velat et Y.Champailler, Paris, Gallimard, "Bibliothèque de la Pléiade », 1961, p. 356 : «C'est ainsi que Jésus veut être prêché, et il dédaigne pour sa parole, aussi bien que pour sa personne, tout ce que les hommes admirent. »

34. [Note de l'Auteur] Comme c'est surtout de Pascal, et de son mot qu'on s'autorise, pour médire de la rhétorique, on ne trouvera pas mauvais que je reproduise quelques lignes de Nicole, dans son Histoire des Provinciales : « Cette lettre [la première] eut tout le succès qu'on pouvait désirer... Elle produisit dans l'esprit de tous l'effet qu'on en attendait. Elle fit connaître combien le genre d'écrire que Montalte avait choisi était propre pour appliquer le monde à cette dispute. On vit qu'il forçait en quelque sorte les plus insensibles et les plus indifférents à s'y intéresser ; qu'il les remuait, qu'il les gagnait par le plaisir; et que, sans avoir pour fin de leur donner un vain divertissement, il les conduisait agréablement à la connaissance de la vérité. » [Note de l'éditeur] Pour la citation originale, on peut se reporter, entre autres, à l'édition de 1735 des Provinciales, contenant la «Préface de Wendrock» où figure ce texte, disponible en ligne: https://books.google.ch/books? $\mathrm{id}=36 \mathrm{gPAAAAQAAJ} \&$ printsec=frontcover\&hl=it\#v=onepage\&q=genre\%20d'\%C3\%A9crire\&f=false.

35. Boileau, Art poétique, Chant I, Euvres complètes, éd. établie et annotée par Françoise Escal, Paris, Gallimard, «Bibliothèque de la Pléiade », 1966, p. 160 : « Enfin Malherbe vint, et le premier en France, / Fit sentir dans les vers une juste cadence / D'un mot mis en sa place enseigna le pouvoir / Et réduisit la muse aux règles du devoir. »

36. Brunetière renvoie sans doute au même chant de l'Art poétique de Boileau, même si la formule exacte est : « juste cadence ».

37. E. Renan, «Préface », L'Histoire du peuple d'Israël [1888], III, Paris, Calmann-Lévy, 1890, p. VI : «On m'a reproché d'avoir souvent, dans le précédent volume, fait les rapprochements entre les antiques événements et les mouvements des temps modernes. Ce n'est pas de ma faute si, dans le présent volume, j'ai encore été amené à blesser, en ce point, la susceptibilité des rhéteurs. »Félix Pyat (1810-1889), journaliste, auteur dramatique et homme politique français, a été une personnalité de la Commune de Paris.

38. M. Du Camp, Théophile Gautier [1883], Paris, Hachette, 1895, p. 8 : «En tout cas, les vers de Gautier partagent avec ceux de Musset une qualité de premier ordre : seuls, de leur époque, ils ne sont point entachés de rhétorique. »

39. Recueil publié pour la première fois en 1836. Voir A. de Lamartine, Jocelyn, Cuvres poétiques complètes, éd. M. F. Guyard, Paris, Gallimard, « Bibliothèque de la Pléiade », 1963, p. 569-788.

40. Recueil publié pour la première fois en 1864. Voir A. de Vigny, Les Destinées, CEuvres complètes, I, éd. F. Germain et A. Jarry, Paris, Gallimard, "Bibliothèque de la Pléiade », 1986, p. 115-166. 
41. T. Gautier, Albertus ou l'âme et le péché. Légende théologique, Paris, Paulin Libraire-Éditeur, 1833, poème repris dans Poésies complètes, Paris, Charpentier, 1845.

42. Poème de Musset publié pour la première fois dans La Revue des deux Mondes en 1833, repris dans les Poésies nouvelles en 1854. Voir A. de Musset, Rolla, Euvres complètes, éd. Ph. Van Tieghem, Paris, Seuil, 1963, p. 139-146.

43. Les vers cités proviennent de Rolla, respectivement I (v. 1-2, p. 139 de l'édition citée) et IV (v. 1-2 et v. 56-57, p. 143 de l'édition citée).

44. J.-L Guez de Balzac, Les Entretiens de feu M. de Balzac, Paris, Augustin Courbé, 1657, p. 164.

45. Sainte-Beuve, Port Royal, I, livre II, éd. M. Leroy, Paris, Gallimard, «Bibliothèque de la Pléiade », 1953, p. 544 : «Balzac, je l'ai dit ailleurs, c'est la prose française qui fait en public, et avec beaucoup d'éclat, sa rhétorique, une double et triple année de rhétorique. »

46. Euvres complètes de Bossuet, vol. IX, éd. F. Lachat, Paris, Louis Vivès, 1862, «Premier Sermon pour le dimanche des Rameaux, sur l'honneur du monde " (p. 572-595) et "Troisième sermon pour le dimanche de la Passion, sur les causes de la haine des hommes contre la vérité » (p. 415-438).

47. J.-J. Rousseau, Le Contrat social, CEuvres complètes, III, éd. B. Gagnebin et M. Raymond, Paris, Gallimard, «Bibliothèque de la Pléiade », 1964. La « Profession de foi du vicaire savoyard » figure dans le livre IV de L'Émile (voir CEuvres complètes, IV, ibid.).

48. F.-R. de Chateaubriand, Le Génie du Christianisme [1802], éd. M. Regard, Paris, Gallimard, « Bibliothèque de la Pléiade », 1978.

49. F. de Lamennais, Essai sur l'indifférence en matière de religion, Paris, Tournachon-Molin \& H. Seguin, 1817.

50. Cicéron, De l'invention [De Inventione], I, II, 3 (trad. G. Achard, Paris, Les Belles Lettres, 1994, p. 58): "Age uero, urbibus constitutis, ut fidem colere et iustitiam retinere discerent et aliis parere sua uoluntate consuescerent ac non modo labores excipiendos communis commodi causa, sed etiam uitam amittendam existimarent, qui tandem fieri potuit, nisi homines ea quae ratione inuenissent eloquentia persuadere potuissent? " "Continuons : Les villes une fois établies, comment aurait-il pu se faire que les gens apprennent à respecter leur parole et à observer la justice, qu'ils s'accoutument à obéir volontairement à d'autres, qu'ils jugent bon non seulement de supporter des fatigues mais même de perdre la vie dans l'intérêt général, s'il n'y avait eu des hommes pour les persuader, grâce à leur éloquence, de ce qu'ils avaient trouvé grâce à leur sagesse ?»

51. "La corruption de ce qu'il y a de meilleur est la pire. » Sous la forme Corruptio optimi pessima, cette formule est «habituellement attribuée à Grégoire le Grand (Moralia in Iob), bien que l'expression semble absente de cette œuvre» (Renzo Tosi, Dictionnaire des sentences latines et grecques, $\mathrm{n}^{\circ}$ 11, Grenoble, Millon, 2010, p. 52-53). Proudhon la rend ainsi : « Il n'y a pas d'iniquité pire que celle du Sage : Corruptio optimi pessima» (De la justice dans la Révolution et dans l'Église, Bruxelles et Leipzig, Schnée, 1860, $4^{\mathrm{e}}$ étude « L’État », p. 28).

52. J. B. Bossuet, CEuvres, op. cit., p. 665-1027, « Discours sur l'histoire universelle à Monseigneur le Dauphin ».

53. J. De Maistre, Soirées de Saint-Petersbourg ou entretiens sur le gouvernement temporel de la Providence, Paris, Rusand, 1822.

54. J. B. Bossuet, CEuvres oratoires, VI, éd. critique de l'Abbé Lebarq, Paris-Lille, 1890-1897, «Sermon sur l'unité de l'église ».

55. J. De Maistre, Du Pape [1819], Paris, Charpentier, 1843 ; Id., L'Église gallicane dans son rapport avec le souverain pontife [1820], Paris, Rusand, 1829.

56. Référence à l'ouvrage de Gustav Gerber, Die Sprache als Kunst, Bromberg, Mittler Verlag, 1871.

57. Pascal, Pensées, pensée $n^{\circ} 41$ [Sellier $n^{\circ}$ 78], «II. Vanité » (dans Euvres complètes, II, op. cit., p. 553). Brunetière déforme légèrement le texte d'origine, qui est le suivant: "Le ton de voix impose aux plus sages, et change un discours et un poème de force. L'affection ou la haine change la justice de face.» 
58. J. B. Massillon, Le Petit Carême [1718], CEuvres choisies de Massillon, Paris, Garnier Frères, 1866.

59. Boutade de Théophile Gautier rapportée par Edmond de Goncourt, quoique dans une version légèrement différente ( j'amène 520 sur une Tête de Turc et je fais des métaphores qui se suivent ») dans sa préface à É. Bergerat, Théophile Gautier : entretiens, souvenirs et correspondance, Paris, Charpentier, 1879, p. IX.

60. [Note de l'Auteur, ses italiques]. "TRISSOTIN : Pour cette grande faim qu'à mes yeux on expose, / Un plat seul de huit vers me semble peu de chose, / Et je pense qu'ici je ne ferai pas mal, / De joindre à l'épigramme ou bien du madrigal. / Le ragoût d'un sonnet qui, chez une princesse, / A passé pour avoir quelque délicatesse, / Il est de sel attique assaisonné partout, / Et vous le trouverez, je crois, d'assez bon goût. " [Note de l'éditeur] La citation provient des Femmes savantes de Molière, acte III, scène 2 .

61. Lettre de Flaubert à Louise Colet datée du 31 mars 1853. Brunetière omet quelques phrases ; voici la citation complète (G. Flaubert, Correspondance, II, éd. J. Bruneau, Paris, Gallimard, «Bibliothèque de la Pléiade ", 1980, p. 289) : « Nous nous étonnons des bonshommes du siècle de Louis XIV, mais ils n'étaient pas des hommes d'énorme génie. On n'a aucun de ces ébahissements, en les lisant, qui vous fassent croire en eux à une nature plus qu'humaine, comme à la lecture d'Homère, de Rabelais, de Shakespeare surtout ; non! Mais quelle conscience ! Comme ils se sont efforcés de trouver [pour] leurs pensées les expressions justes! Quel travail ! quelles ratures ! Comme ils se consultaient les uns les autres. Comme ils savaient le latin ! Comme ils lisaient lentement ! Aussi toute leur idée y est, la forme est pleine, bourrée \& garnie de choses jusqu'à la faire craquer. "

62. Fénelon, «Lettre à l'Académie française», Euvres, II, éd. J. Lebrun, Paris, Gallimard, «Bibliothèque de la Pléiade », 1997.

63. Voir Littré, s. v. muet, $12^{\mathrm{e}}$ : « À la muette, loc. adv. Sans faire de bruit. »

64. C. F. de Vaugelas, "Préface", Remarques sur la langue françoise: utiles à ceux qui veulent bien parler et bien escrire, Paris, Vve J. Camusat et P. Le Petit, 1647, n.p.

\section{AUTEURS}

\section{FERDINAND BRUNETIÈRE}

1849-1906 